\title{
Lowest Landau-level description of a Bose-Einstein condensate in a rapidly rotating anisotropic trap
}

\author{
Alexander L. Fetter \\ Departments of Physics and Applied Physics, Stanford University, Stanford, CA 94305-4045, USA
}

(Dated: September 14, 2018)

\begin{abstract}
A rapidly rotating Bose-Einstein condensate in a symmetric two-dimensional trap can be described with the lowest Landau-level set of states. In this case, the condensate wave function $\psi(x, y)$ is a Gaussian function of $r^{2}=x^{2}+y^{2}$, multiplied by an analytic function $P(z)$ of the single complex variable $z=x+i y$; the zeros of $P(z)$ denote the positions of the vortices. Here, a similar description is used for a rapidly rotating anisotropic two-dimensional trap with arbitrary anisotropy $\left(\omega_{x} / \omega_{y} \leq 1\right)$. The corresponding condensate wave function $\psi(x, y)$ has the form of a complex anisotropic Gaussian with a phase proportional to $x y$, multiplied by an analytic function $P(\zeta)$, where $\zeta \propto x+i \beta_{-} y$ and $0 \leq \beta_{-} \leq 1$ is a real parameter that depends on the trap anisotropy and the rotation frequency. The zeros of $P(\zeta)$ again fix the locations of the vortices. Within the set of lowest Landau-level states at zero temperature, an anisotropic parabolic density profile provides an absolute minimum for the energy, with the vortex density decreasing slowly and anisotropically away from the trap center.
\end{abstract}

PACS numbers: $03.75 . \mathrm{Hh}, 05.30 . \mathrm{Jp}, 67.40 . \mathrm{Db}$

\section{INTRODUCTION}

The experimental creation of rapidly rotating Bose-Einstein condensates (BEC) generally involves anisotropic rotating trap potentials [1, 2, 3], yet most theoretical analyses of such systems have relied on an isotropic trap [4, 5, 6, 7, 8, 9]. As emphasized by Ho [4], the low-lying states in a symmetric two-dimensional trap are closely analogous to those in the lowest Landau level for a charged particle in a uniform magnetic field. This analogy allows a simplified description in the limit that the rotation frequency $\Omega$ approaches the frequency $\omega_{0}$ of the symmetric confining trap. If the typical interaction energy is small compared to the spacing $\approx 2 \hbar \omega_{0}$ between adjacent Landau levels, then the condensate wave function $\psi$ of the interacting rotating BEC can be constructed as a linear combination of the lowest Landau-level (LLL) states. The $n$th such state is simply proportional to $z^{n}$, where $z=x+i y$, multiplied by the ground-state Gaussian. It follows that such a linear combination involves an analytic function of $z$ that is usually approximated by a polynomial $P(z) \propto \prod_{j}\left(z-z_{j}\right)$, where the zeros $z_{j}$ of the polynomial represent the positions of the vortices in the two-dimensional condensate. If the vortex density is strictly uniform, then the overall density profile is also Gaussian with an effective condensate radius that grows and ultimately diverges as $\Omega \rightarrow \omega_{0}[\underline{4}$. In fact, this system can lower its energy by slightly reducing the vortex density near the outer edge of the condensate, and the actual density profile has a quadratic shape (an inverted parabola) [5, 6, 67, [] ], as shown by both analytical and numerical studies.

The quantum-mechanical problem of a particle in a rotating two-dimensional anisotropic harmonic trap is exactly soluble 10, 11, 12], although the corresponding eigenstates have not been discussed previously in full detail. We here amplify Valatin's description [10] to construct the anisotropic analogs of the LLL states. Each such state $\varphi_{n 0}(x, y)$ involves the anisotropic complex Gaussian ground-state eigenfunction $\varphi_{00}(x, y)$, multiplied by a polynomial $p_{n}(\zeta)$, where $\zeta \propto x+i \beta_{-} y$ is a single "stretched" complex variable, and $0 \leq \beta_{-} \leq 1$ is a real parameter that depends on the trap anisotropy and the rotation frequency. Thus a linear combination of these LLL states for an anisotropic trap again involves an analytic function of the single complex variable $\zeta$ (apart from the common overall factor $\varphi_{00}$ ). The corresponding zeros again represent the positions of the vortices, now in the rotating anisotropic BEC.

Section II focuses on the eigenstates of the single-particle Hamiltonian $H_{0}$ for a rotating anisotropic harmonic potential, starting with the classical trajectories and then obtaining the explicit form of the low-lying quantum mechanical states $\varphi_{n 0}$ that are the analogs of the lowest Landau-level states for a charged particle in a magnetic field. As in that case, the expectation value of both $H_{0}$ and the angular momentum $L_{z}$ in the lowest Landau level can be reduced to corresponding expectation values of $x^{2}$ and $y^{2}$. The interacting dilute Bose-Einstein gas in this rapidly rotating anisotropic trap is treated in Sec. III. For a trial state constructed as a linear combination of $\varphi_{n 0}(x, y)$, an anisotropic parabolic density profile provides the absolute minimum of the energy. The density of vortices is constant near the center but decreases slowly toward the edge of the condensate. Section IV contains a discussion and suggestions for additional study. 


\section{SINGLE-PARTICLE EIGENSTATES}

Consider a particle of mass $m$ in an anisotropic two-dimensional harmonic potential (for definiteness, I assume oscillator frequencies $\omega_{x} \leq \omega_{y}$ ) that rotates uniformly at an angular velocity $\boldsymbol{\Omega}=\Omega \hat{\boldsymbol{z}}$ perpendicular to the plane of the motion. In the rotating frame, this potential is time-independent, with the Hamiltonian

$$
H_{0}=\frac{p_{x}^{2}+p_{y}^{2}}{2 m}+\frac{1}{2} m\left(\omega_{x}^{2} x^{2}+\omega_{y}^{2} y^{2}\right)-\Omega\left(x p_{y}-y p_{x}\right),
$$

where the last factor involves the angular momentum $L_{z}=x p_{y}-y p_{x}$. If $\omega_{x}<\omega_{y}$, the centrifugal force preferentially expands the condensate along the $x$ axis. Although this case $\left(\omega_{x}<\omega_{y}\right)$ is of principal interest here, it will also be valuable to see how the more familiar symmetric case emerges in the limit $\omega_{x}=\omega_{y}=\omega_{0}$.

\section{A. Classical dynamical trajectories}

The normal modes of Eq. (11) are readily determined to have the frequencies [10, 11, 12, 13, 14]

$$
\omega_{ \pm}^{2}=\omega_{\perp}^{2}+\Omega^{2} \mp \sqrt{\frac{1}{4}\left(\omega_{y}^{2}-\omega_{x}^{2}\right)^{2}+4 \omega_{\perp}^{2} \Omega^{2}}
$$

where $\omega_{\perp}^{2}=\frac{1}{2}\left(\omega_{x}^{2}+\omega_{y}^{2}\right)$ is the mean-squared oscillator frequency. This general result contains several important limits.

1. In the symmetric case $\omega_{x}=\omega_{y}=\omega_{0}$, the plus (minus) modes have frequencies $\omega_{ \pm}=\omega_{0} \mp \Omega$. Specifically, the plus mode with frequency $\omega_{+}=\omega_{0}-\Omega$ has a reduced frequency when viewed from the rotating frame (as is evident physically) and a positive angular momentum (which explains the notation). Correspondingly, the minus mode has an increased frequency $\omega_{-}=\omega_{0}+\Omega$ and a negative angular momentum.

2. If $\omega_{x}<\omega_{y}$, then the modes are nondegenerate even for $\Omega=0$, when they reduce to $\omega_{+}=\omega_{x}$ and $\omega_{-}=\omega_{y}$.

3. For an anisotropic trap $\left(\omega_{x}<\omega_{y}\right)$ and rapid rotation with $\delta=1-\Omega / \omega_{x} \rightarrow 0^{+}$, the plus normal-mode frequency vanishes, with

$$
\omega_{+}^{2} \approx \frac{2 \omega_{x}^{2}\left(\omega_{y}^{2}-\omega_{x}^{2}\right)}{3 \omega_{x}^{2}+\omega_{y}^{2}} \delta
$$

Thus $-\partial \omega_{+} / \partial \Omega$ diverges for small $\delta$ like $\delta^{-1 / 2}$. In contrast, the minus normal-mode frequency remains finite at $\delta=0$, with

$$
\omega_{-}^{2} \approx 3 \omega_{x}^{2}+\omega_{y}^{2}
$$

4. In the case of a rapidly rotating nearly symmetric trap with two small parameters $[12] \delta=1-\Omega / \omega_{x}$ and $\eta=\omega_{y} / \omega_{x}-1$, these eigenfrequencies simplify to

$$
\frac{\omega_{+}}{\omega_{x}} \approx \sqrt{\delta(\eta+\delta)}, \quad \frac{\omega_{-}}{\omega_{x}} \approx 2+\frac{1}{2} \eta-\delta .
$$

Note the sensitivity of $\omega_{+}$in Eq. (5) to the order of limiting procedures: (i) first $\eta=0\left(\right.$ namely $\left.\omega_{x}=\omega_{y}=\omega_{0}\right)$ and then $\delta \rightarrow 0$ or (ii) $\delta \rightarrow 0$ (namely $\Omega \rightarrow \omega_{x}$ ) at fixed $\omega_{x}<\omega_{y}$. For a symmetric trap, the plus frequency $\omega_{+}$vanishes linearly with the small parameter $\delta$; in contrast, for the anisotropic trap with $\omega_{x}<\omega_{y}$, the plus frequency vanishes like $\sqrt{\delta}$, with a coefficient proportional to $\sqrt{\eta}$.

Figure $1(\mathrm{a})$ shows the two different normal-mode frequencies $\omega_{ \pm}$(normalized to $\omega_{x}$ ) as functions of $\Omega / \omega_{x}$ for the typical anisotropy $\omega_{y} / \omega_{x}=1.2$.

For a symmetric trap with $\omega_{x}=\omega_{y}=\omega_{0}$, the plus (minus) normal modes with frequencies $\omega_{ \pm}=\omega_{0} \mp \Omega$ have counterclockwise (clockwise) circular orbits with positive (negative) helicity. To understand the physics of the two normal modes in the more general anisotropic case $\omega_{x}<\omega_{y}$, consider first the motion in the plus mode [10]. It has the form

$$
x_{+}(t)=x_{0} e^{-i \omega_{+} t}, \quad y_{+}(t)=i \beta_{+} x_{0} e^{-i \omega_{+} t},
$$



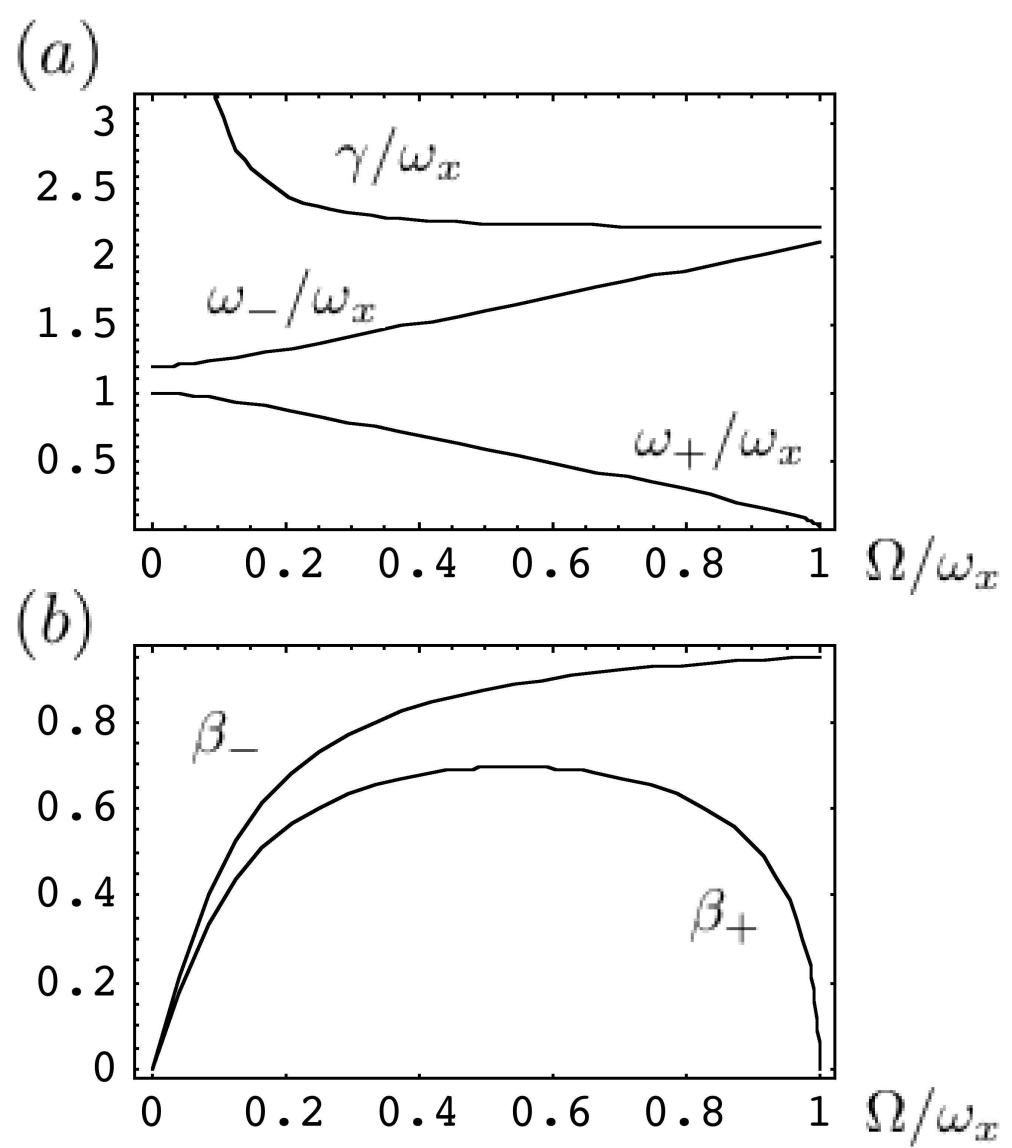

FIG. 1: Behavior of relevant dimensionless quantities as a function of dimensionless rotation speed $\Omega / \omega_{x}$ for the typical anisotropy $\omega_{y} / \omega_{x}=1.2$. (a) dimensionless normal mode frequencies $\omega_{+} / \omega_{x}$ and $\omega_{-} / \omega_{x}$ and dimensionless auxiliary frequency $\gamma / \omega_{x} ;$ (b) dimensionless polarization parameters $\beta_{+}$and $\beta_{-}$.

where $0 \leq \beta_{+} \leq 1$ is a real non-negative dimensionless parameter and $x_{0}$ is an arbitrary amplitude. A detailed analysis shows that the parameter $\beta_{+}$has two alternative representations

$$
\beta_{+}=\frac{\omega_{x}^{2}-\omega_{+}^{2}-\Omega^{2}}{2 \Omega \omega_{+}}=\frac{2 \Omega \omega_{+}}{\omega_{y}^{2}-\omega_{+}^{2}-\Omega^{2}} .
$$

In the rotating frame, the plus orbit is an ellipse with major axis oriented along $\hat{\boldsymbol{x}}$; the polarization parameter $\beta_{+}$ gives the ratio of the minor to major axes. The second form of Eq. (7) shows that $\omega_{+} / \beta_{+} \approx\left(\omega_{y}^{2}-\omega_{x}^{2}\right) /\left(2 \omega_{x}\right)$ for $\delta \rightarrow 0$, so that $\omega_{+}$and $\beta_{+}$both vanish in this limit like $\sqrt{\delta}$. Physically, this behavior reflects the rotation-induced cancellation of the harmonic confinement in the $\hat{\boldsymbol{x}}$ direction; the orbit then becomes linearly polarized as $\Omega \rightarrow \omega_{x}$. The plus motion is counterclockwise, with positive helicity and positive angular momentum.

Similarly, the orbit for the minus mode has the parametric representation

$$
x_{-}(t)=i \beta_{-} y_{0} e^{-i \omega_{-} t}, \quad y_{-}(t)=y_{0} e^{-i \omega_{-} t},
$$

where $y_{0}$ is an arbitrary amplitude and $0 \leq \beta_{-} \leq 1$ is a real non-negative parameter with two alternative representations [10]

$$
\beta_{-}=\frac{\omega_{-}^{2}-\omega_{y}^{2}+\Omega^{2}}{2 \Omega \omega_{-}}=\frac{2 \Omega \omega_{-}}{\omega_{-}^{2}-\omega_{x}^{2}+\Omega^{2}} .
$$

Unlike $\beta_{+}$, these relations show that $\beta_{-}$has a nonzero limit $\beta_{-} \approx 2 \omega_{x} /\left(3 \omega_{x}^{2}+\omega_{y}^{2}\right)^{1 / 2} \leq 1$ as $\delta \rightarrow 0$. In the rotating frame, the minus orbit is an ellipse with major axis oriented along $\hat{\boldsymbol{y}}$; the polarization parameter $\beta_{-}$gives the ratio of the minor to major axes. The minus motion is clockwise, with negative helicity and negative angular momentum. 
It is again instructive to specialize these results to the case of a rapidly rotating nearly symmetric trap [12], making use of the small parameters $\delta=1-\Omega / \omega_{x}$ and $\eta=\omega_{y} / \omega_{x}-1$. A straightforward expansion yields the approximate polarization parameters

$$
\beta_{+} \approx \sqrt{\frac{\delta}{\eta+\delta}}, \quad \beta_{-} \approx 1-\frac{1}{4} \eta
$$

Although $\beta_{-}$varies smoothly in this limit, the corresponding polarization $\beta_{+}$for the positive mode has a singular behavior that depends on the relative magnitude of the two small parameters. More generally, $\beta_{+}$and $\beta_{-}$both have the value 1 in the limit of a symmetric trap, independent of $\Omega$, since the resulting motion is circularly polarized. Figure 1 (b) shows the dependence of the two parameters $\beta_{ \pm}$on $\Omega / \omega_{x}$ for an anisotropy $\omega_{y} / \omega_{x}=1.2$. Note the singular slope of $\omega_{+}$and $\beta_{+}$near the upper limit.

\section{B. Bogoliubov canonical transformation to diagonal Hamiltonian}

The structure of $H_{0}$ in Eq. (11) is unusual because the term $\Omega L_{z}=\Omega\left(y p_{x}-x p_{y}\right)$ couples the otherwise independent $x$ and $y$ motions. This situation can be clarified by introducing the conventional ladder operators [15]

$$
a_{x}=\frac{1}{\sqrt{2}}\left(\frac{x}{d_{x}}+i \frac{d_{x} p_{x}}{\hbar}\right), \quad a_{x}^{\dagger}=\frac{1}{\sqrt{2}}\left(\frac{x}{d_{x}}-i \frac{d_{x} p_{x}}{\hbar}\right),
$$

where $d_{x}=\sqrt{\hbar /\left(m \omega_{x}\right)}$, and similarly for $a_{y}$ and $a_{y}^{\dagger}$. With these operators, it is straightforward to see that the term $\Omega L_{z}$ is proportional to [1]

$$
i \Omega\left[\left(\omega_{y}+\omega_{x}\right)\left(a_{x}^{\dagger} a_{y}-a_{y}^{\dagger} a_{x}\right)+\left(\omega_{y}-\omega_{x}\right)\left(a_{x} a_{y}-a_{y}^{\dagger} a_{x}^{\dagger}\right)\right] .
$$

The first term is "diagonal" in the creation and annihilation operators, but the second is "off-diagonal," similar to Bogoliubov's approximate Hamiltonian for a dilute Bose-Einstein gas [16]. Unfortunately, a direct diagonalization based on these "particle" operators involves considerable algebraic complexity [11, 12].

Thus, it is preferable to return to the original single-particle Hamiltonian in Eq. (II). Since $H_{0}$ is quadratic in the coordinates and momenta, it can be diagonalized with a canonical transformation to new variables that obey the same Poisson brackets (in the classical case) or the same commutators (in the quantum case). Specifically, I follow Valatin [10] and introduce the generating function

$$
S\left(x, y ; Q_{+}, Q_{-}\right)=-m \gamma\left[\lambda_{+} \lambda_{-} Q_{+} Q_{-}+\frac{1}{2}\left(\lambda_{+}^{2}+\lambda_{-}^{2}\right) x y-\lambda_{+} Q_{+} y-\lambda_{-} Q_{-} x\right] .
$$

Here, $Q_{ \pm}$are new canonical coordinates, $\lambda_{ \pm}$are dimensionless constants given by

$$
\lambda_{ \pm}^{2}=\frac{\omega_{ \pm}}{\mu_{ \pm}}, \quad \text { with } \quad \mu_{ \pm}=\omega_{ \pm}+\beta_{+} \beta_{-} \omega_{\mp},
$$

and $\gamma=\mu_{+} / \beta_{+}=\mu_{-} / \beta_{-}$has the dimensions of a frequency. It follows from Eqs. (21), (7), (9) and (14) that $\gamma$ has various equivalent representations

$$
\begin{aligned}
\gamma & =\frac{\omega_{+}}{\beta_{+}}+\omega_{-} \beta_{-}=\frac{\omega_{-}}{\beta_{-}}+\omega_{+} \beta_{+} \\
& =\frac{\omega_{-}^{2}-\omega_{+}^{2}}{2 \Omega}=\frac{\sqrt{\frac{1}{4}\left(\omega_{y}^{2}-\omega_{x}^{2}\right)^{2}+4 \omega_{\perp}^{2} \Omega^{2}}}{\Omega} .
\end{aligned}
$$

For a symmetric trap with $\omega_{x}=\omega_{y}=\omega_{0}$, this frequency reduces to $\gamma=2 \omega_{0}$ for all $\Omega$. In contrast, for an anisotropic trap, $\gamma$ diverges as $\Omega$ becomes small and approaches the value $\approx\left(3 \omega_{x}^{2}+\omega_{y}^{2}\right) /\left(2 \omega_{x}\right)>2 \omega_{x}$ for $\Omega \rightarrow \omega_{x}$. Figure 1(a) shows the normalized parameter $\gamma / \omega_{x}$ as a function of $\Omega / \omega_{x}$ for $\omega_{y} / \omega_{x}=1.2$.

According to the general theory of classical Hamiltonian dynamics [17], any function like $S\left(x, y ; Q_{+}, Q_{-}\right)$that depends on both the old and new coordinates will automatically generate a canonical transformation from old canonical variables to new canonical variables, with the corresponding momentum variables given by

$$
\begin{array}{cc}
p_{x}=\frac{\partial S}{\partial x}, & p_{y}=\frac{\partial S}{\partial y} \\
P_{+}=-\frac{\partial S}{\partial Q_{+}}, & P_{-}=-\frac{\partial S}{\partial Q_{-}} .
\end{array}
$$


The first set of equations immediately yields the relations

$$
\begin{aligned}
& Q_{+}=\left(\frac{\lambda_{+}^{2}+\lambda_{-}^{2}}{2 \lambda_{+}}\right) x+\frac{p_{y}}{m \gamma \lambda_{+}} \\
& Q_{-}=\left(\frac{\lambda_{+}^{2}+\lambda_{-}^{2}}{2 \lambda_{-}}\right) y+\frac{p_{x}}{m \gamma \lambda_{-}}
\end{aligned}
$$

that express the new coordinates $Q_{ \pm}$as linear combinations of the original coordinates and momenta. Similarly, the second set of equations can be used to find the corresponding relations for the new momenta $P_{ \pm}$

$$
\begin{aligned}
& P_{+}=m \gamma \lambda_{+}\left(\frac{\lambda_{+}^{2}+\lambda_{-}^{2}}{2}-1\right) y+\lambda_{+} p_{x}, \\
& P_{-}=m \gamma \lambda_{-}\left(\frac{\lambda_{+}^{2}+\lambda_{-}^{2}}{2}-1\right) x+\lambda_{-} p_{y} .
\end{aligned}
$$

For future reference, note the following alternative relations

$$
\begin{aligned}
& x=\lambda_{+} Q_{+}-\frac{P_{-}}{m \gamma \lambda_{-}}, \\
& y=\lambda_{-} Q_{-}-\frac{P_{+}}{m \gamma \lambda_{+}}
\end{aligned}
$$

they express the original coordinates in terms of the new canonical variables and will be valuable in the subsequent analysis.

It is now straightforward to verify that the Hamiltonian has the following simple diagonal form when expressed in the new canonical variables

$$
H_{0}=\frac{P_{+}^{2}}{2 m}+\frac{1}{2} m \omega_{+}^{2} Q_{+}^{2}+\frac{P_{-}^{2}}{2 m}+\frac{1}{2} m \omega_{-}^{2} Q_{-}^{2} .
$$

One strategy is to substitute Eqs. (17) and (18) directly into (20), which eventually reproduces the original Eq. (11). This new Hamiltonian (20) has the great advantage of immediately providing a quantum description of two independent harmonic oscillators with mass $m$ and frequencies $\omega_{ \pm}$. The corresponding quantum-mechanical annihilation operators $\alpha_{ \pm}$and creation operators $\alpha_{ \pm}^{\dagger}$ follow from general quantum theory [15]

$$
\alpha_{ \pm}=\frac{1}{\sqrt{2}}\left(\frac{Q_{ \pm}}{d_{ \pm}}+i \frac{d_{ \pm} P_{ \pm}}{\hbar}\right), \quad \alpha_{ \pm}^{\dagger}=\frac{1}{\sqrt{2}}\left(\frac{Q_{ \pm}}{d_{ \pm}}-i \frac{d_{ \pm} P_{ \pm}}{\hbar}\right)
$$

where $d_{ \pm}=\sqrt{\hbar /\left(m \omega_{ \pm}\right)}$are the oscillator lengths for the two separate modes. These operators obey the usual commutation relations $\left[\alpha_{ \pm}, \alpha_{ \pm}^{\dagger}\right]=1$ (all other commutators vanish). Note that $d_{+}$diverges as $\Omega \rightarrow \omega_{x}$, whereas $d_{-}$ remains finite in the same limit. In terms of these operators, the Hamiltonian takes the form [1]

$$
H_{0}=\frac{1}{2} \hbar \omega_{+}\left(\alpha_{+}^{\dagger} \alpha_{+}+\alpha_{+} \alpha_{+}^{\dagger}\right)+\frac{1}{2} \hbar \omega_{-}\left(\alpha_{-}^{\dagger} \alpha_{-}+\alpha_{-} \alpha_{-}^{\dagger}\right) .
$$

\section{Lowest Landau-level single-particle states for rotating anisotropic trap}

The single-particle ground state $\varphi_{00}$ is given by the prescription $\alpha_{ \pm} \varphi_{00}=0$, which leads to the explicit representation

$$
\varphi_{00} \propto \exp \left(-\frac{Q_{+}^{2}}{2 d_{+}^{2}}-\frac{Q_{-}^{2}}{2 d_{-}^{2}}\right)
$$

as a Gaussian function of the two new coordinates $Q_{ \pm}$. Correspondingly, the complete set of normalized single-particle states $\varphi_{n_{+} n_{-}}$is specified by two non-negative integers $n_{ \pm}$

$$
\varphi_{n_{+} n_{-}}=\frac{\left(\alpha_{+}^{\dagger}\right)^{n_{+}}}{\sqrt{n_{+} !}} \frac{\left(\alpha_{-}^{\dagger}\right)^{n_{-}}}{\sqrt{n_{-} !}} \varphi_{00} .
$$


Here, the eigenstate state $\varphi_{n_{+} n_{-}}$has $n_{+}\left(n_{-}\right)$quanta with frequency $\omega_{+}\left(\omega_{-}\right)$; the energy eigenvalue is

$$
\epsilon_{n_{+} n_{-}}=\hbar \omega_{+}\left(n_{+}+\frac{1}{2}\right)+\hbar \omega_{-}\left(n_{-}+\frac{1}{2}\right) .
$$

This eigenstate $\varphi_{n_{+} n_{-}}$has an angular momentum [1]

$$
L_{n_{+} n_{-}}=-\frac{\partial \epsilon_{n_{+} n_{-}}}{\partial \Omega}=-\hbar \frac{\partial \omega_{+}}{\partial \Omega}\left(n_{+}+\frac{1}{2}\right)-\hbar \frac{\partial \omega_{-}}{\partial \Omega}\left(n_{-}+\frac{1}{2}\right) .
$$

Since $\partial \omega_{+} / \partial \Omega$ is negative ( $\partial \omega_{-} / \partial \Omega$ is positive), this result confirms that the plus (minus) mode has positive (negative) angular momentum. For the special case of a symmetric trap, the detailed form of these eigenstates is well known 18, 19 .

It is important to re-express the ground-state wave function $\varphi_{00}$ in terms of the original canonical coordinates $x$ and $y$. Valatin [10] uses the generating function $S\left(x, y ; Q_{+}, Q_{-}\right)$in Eq. (13) to obtain the explicit (factorized) expression

$$
\varphi_{00}(x, y) \propto \exp \left[-\frac{m \gamma\left(\beta_{+} x^{2}+\beta_{-} y^{2}\right)}{2 \hbar\left(1+\beta_{+} \beta_{-}\right)}\right] \exp \left\{i \frac{m x y}{\hbar}\left[\frac{\gamma}{1+\beta_{+} \beta_{-}}-\frac{1}{2}\left(\frac{\omega_{+}}{\beta_{+}}+\frac{\omega_{-}}{\beta_{-}}\right)\right]\right\} .
$$

Each of these two factors has an interesting structure.

1. The first factor is a real anisotropic Gaussian with characteristic lengths $a_{x}$ and $a_{y}$ given by

$$
a_{x}^{2}=\frac{1+\beta_{+} \beta_{-}}{\beta_{+}} \frac{\hbar}{m \gamma}, \quad a_{y}^{2}=\frac{1+\beta_{+} \beta_{-}}{\beta_{-}} \frac{\hbar}{m \gamma} .
$$

In the limit of a symmetric trap, this Gaussian ground state becomes $\varphi_{00}(x, y) \propto \exp \left[-m \omega_{0}\left(x^{2}+y^{2}\right) /(2 \hbar)\right]$, with the expected oscillator length $d_{0}=\sqrt{\hbar /\left(m \omega_{0}\right)}$. More generally, the ground-state density for a rotating anisotropic trap is an anisotropic Gaussian, given by the corresponding normalized wave function

$$
\left|\varphi_{00}(x, y)\right|^{2}=\frac{1}{\pi a_{x} a_{y}} \exp \left(-\frac{x^{2}}{a_{x}^{2}}-\frac{y^{2}}{a_{y}^{2}}\right) .
$$

For a rapidly rotating anisotropic trap $\left(\omega_{x}<\omega_{y}\right.$ and $\left.\delta=1-\Omega / \omega_{x} \rightarrow 0\right)$, the length $a_{x}$ diverges because $\beta_{+} \rightarrow 0$, but $a_{y}$ remains finite. In this limit, the ground-state density becomes an essentially one-dimensional strip with Gaussian transverse profile and finite width $a_{y}[20,21]$.

2. The second factor of $\varphi_{00}$ involves a complex phase proportional to $x y$, which reflects the irrotational flow induced by the rotating anisotropic trap $22,23,24]$. The factor in square brackets (the coefficient of $i m x y / \hbar$ ) has a rather intricate structure. It vanishes for a symmetric trap, because $\beta_{ \pm}=1$ and $\omega_{+}+\omega_{-}=\gamma=2 \omega_{0}$. It also vanishes for a stationary anisotropic trap, but this limit requires a detailed analysis because each term separately diverges as $\Omega \rightarrow 0$. This phase will be seen to play an essential role in the following construction of the lowest Landau-level states.

With Eqs. (17) and (18), the operators $\alpha_{ \pm}$and $\alpha_{ \pm}^{\dagger}$ defined in Eqs. (21) are readily expressed in terms of the original coordinates $x, y$ and momenta $p_{x}, p_{y}$. It is not hard to verify explicitly that $\alpha_{ \pm} \varphi_{00}(x, y)=0$. The more interesting question is the form of the low-lying states $\varphi_{n 0}(x, y)$, which are the analogs of the lowest Landau-level states but now for an anisotropic rotating trap. In this case, the state $\varphi_{n 0}$ has $n$ quanta of the plus mode (whose frequency $\omega_{+}$ becomes small $\propto \sqrt{\delta}$ for $\delta=1-\Omega / \omega_{x} \rightarrow 0$ ), and zero quanta of the minus mode (which has a finite frequency $\omega_{-}$in the same limit).

The basic relation $\alpha_{+} \varphi_{00}=0$ implies that $\left(Q_{+} / \sqrt{2} d_{+}\right) \varphi_{00}=-i\left(d_{+} P_{+} / \sqrt{2} \hbar\right) \varphi_{00}$. Thus $\alpha_{+}^{\dagger} \varphi_{00}=$ $\left(\sqrt{2} Q_{+} / d_{+}\right) \varphi_{00}=-i\left(\sqrt{2} d_{+} P_{+} / \hbar\right) \varphi_{00}$, and a straightforward calculation yields

$$
\varphi_{10}(x, y)=\alpha_{+}^{\dagger} \varphi_{00}(x, y)=\zeta \varphi_{00}(x, y)
$$

where $\varphi_{00}$ is the normalized ground state, and

$$
\begin{aligned}
\zeta & =\frac{\sqrt{2}\left(x+i \beta_{-} y\right)}{d_{+} \lambda_{+}\left(1+\beta_{+} \beta_{-}\right)}=\sqrt{\frac{2 m \gamma \beta_{+}}{\hbar}} \frac{x+i \beta_{-} y}{1+\beta_{+} \beta_{-}} \\
& =\sqrt{\frac{2}{1+\beta_{+} \beta_{-}}} \frac{x+i \beta_{-} y}{a_{x}}
\end{aligned}
$$


is a dimensionless complex variable involving a "stretched" combination $x+i \beta_{-} y$. As shown in Fig. 1(b), the polarization parameter $\beta_{-}$is real and less that 1 in the limit of rapidly rotating anisotropic trap. This complex variable reduces to [4] $\zeta=(x+i y) / d_{0}$ for a rotating symmetric trap, where $d_{0}=\sqrt{\hbar /\left(m \omega_{0}\right)}$. In the more general case of a rotating anisotropic trap, the characteristic length that appears in (31) is essentially $a_{x}$ from Eq. (28), apart from the common factor $\sqrt{\frac{1}{2}\left(1+\beta_{+} \beta_{-}\right)}$and the additional factor $\beta_{-}$for $y$; both these factors remain finite as $\Omega \rightarrow \omega_{x}$. This quasi-isotropic behavior for $\zeta$ is very different from the anisotropy seen in the two lengths $a_{x}$ and $a_{y}$ that determine the $x$ and $y$ structure of the ground-state density $\left|\varphi_{00}\right|^{2}$.

The higher states within the lowest Landau level have a similar structure. For example, $\varphi_{20}=\left(\alpha_{+}^{\dagger}\right)^{2} \varphi_{00} / \sqrt{2}$ can be written as

$$
\begin{aligned}
\varphi_{20}(x, y) & =\frac{\alpha_{+}^{\dagger}}{\sqrt{2}} \varphi_{10}(x, y)=\frac{\alpha_{+}^{\dagger}}{\sqrt{2}} \zeta \varphi_{00}(x, y) \\
& =\frac{1}{\sqrt{2}}\left(-\left[\zeta, \alpha_{+}^{\dagger}\right]+\zeta^{2}\right) \varphi_{00}(x, y) .
\end{aligned}
$$

The commutator is readily evaluated with Eqs. (21), (17), (18) and (31), yielding

$$
\left[\zeta, \alpha_{+}^{\dagger}\right]=\frac{1-\beta_{+} \beta_{-}}{1+\beta_{+} \beta_{-}} \equiv c,
$$

which defines the constant $c$ (it depends on the trap frequencies $\omega_{x}, \omega_{y}$ and the rotation speed $\Omega$ ). In general, $0 \leq c \leq 1$, but it vanishes identically for a symmetric trap since $\beta_{ \pm}=1$ in this case. Thus $\varphi_{20}=\left(\zeta^{2}-c\right) \varphi_{00} / \sqrt{2}$, namely an even polynomial in $\zeta$ times the complex Gaussian ground state $\varphi_{00}$. As a check on this analysis, note that $\left[\alpha_{+}, \alpha_{+}^{\dagger}\right] \varphi_{00}=\varphi_{00}$, and direct calculation verifies that $\left[\alpha_{+}, \zeta\right]=1$.

The general lowest Landau-level state follows from similar arguments [it is essential here that the commutator (33) is a pure number, independent of $x$ and $y$ ]

$$
\varphi_{n 0}(x, y)=\frac{1}{\sqrt{n !}} p_{n}(\zeta) \varphi_{00}(x, y)
$$

where $p_{n}(\zeta)$ is a polynomial of order $n$ that obeys the symmetry condition $p_{n}(-\zeta)=(-1)^{n} p_{n}(\zeta)$. These polynomials are easily obtained recursively with the relation

$$
p_{n+1}(\zeta)=\zeta p_{n}(\zeta)-c \frac{d p_{n}(\zeta)}{d \zeta}
$$

with the first few given explicitly as $p_{0}=1, p_{1}=\zeta, p_{2}=\zeta^{2}-c, p_{3}=\zeta^{3}-3 c \zeta, \cdots$. The Hermite polynomials $H_{n}(x)$ obey a similar recursion relation [25] $H_{n+1}(x)=2 x H_{n}(x)-H_{n}^{\prime}(x)$. Direct comparison shows that

$$
p_{n}(\zeta)=\left(\frac{c}{2}\right)^{n / 2} H_{n}\left(\frac{\zeta}{\sqrt{2 c}}\right)
$$

which readily reproduces the explicit forms given above for small $n=0, \cdots, 3$. Oktel $[12$ obtained an analogous but less general result in the special limit of small anisotropy and rapid rotation. For a symmetric trap (with $\beta_{ \pm}=1$ and $c=0)$, it follows directly that $p_{n}(\zeta)$ reduces to the $n$th power of $(x+i y) / d_{0}$.

\section{Expectation value of single-particle $H_{0}$ for general lowest Landau-level state}

Let $\psi_{L L L}=\sum_{n} c_{n} \varphi_{n 0}$ be a general linear combination of lowest Landau-level states $\left\{\varphi_{n 0}\right\}$, normalized with the condition $\int d x d y\left|\psi_{L L L}\right|^{2}=1$. The expectation value of $H_{0}$ in Eq. (10) is given by the equivalent operators in (22)

$$
\left\langle H_{0}\right\rangle=\int d x d y \psi_{L L L}^{*}\left[\frac{1}{2} \hbar \omega_{+}\left(\alpha_{+}^{\dagger} \alpha_{+}+\alpha_{+} \alpha_{+}^{\dagger}\right)+\frac{1}{2} \hbar \omega_{-}\left(\alpha_{-}^{\dagger} \alpha_{-}+\alpha_{-} \alpha_{-}^{\dagger}\right)\right] \psi_{L L L},
$$

where the angular brackets denote the expectation value with the state $\psi_{L L L}$. Since $\alpha_{-} \psi_{L L L}$ vanishes (by construction), this quantity reduces to

$$
\left\langle H_{0}\right\rangle=\frac{1}{2} \hbar \omega_{-}+\frac{1}{2} \hbar \omega_{+} \int d x d y \psi_{L L L}^{*}\left(\alpha_{+}^{\dagger} \alpha_{+}+\alpha_{+} \alpha_{+}^{\dagger}\right) \psi_{L L L},
$$


where the first term is just the zero-point energy of the unoccupied minus mode. For a symmetric trap, this expectation value is readily expressed in terms of the expectation value $\left\langle x^{2}+y^{2}\right\rangle$ [4, 8]. As shown below, a similar but more intricate result holds for the rotating anisotropic trap.

It is convenient to start from Eqs. (19) that express $x$ and $y$ in terms of the new canonical variables $Q_{ \pm}$and $P_{ \pm}$. In turn, these operators are simply linear combinations of the corresponding oscillator variables $\alpha_{ \pm}^{\dagger}$ and $\alpha_{ \pm}$, as follows from (21). For example,

$$
x=\frac{d_{+} \lambda_{+}}{\sqrt{2}}\left(\alpha_{+}+\alpha_{+}^{\dagger}\right)-\frac{\hbar}{\sqrt{2} i d_{-} \lambda_{-} m \gamma}\left(\alpha_{-}-\alpha_{-}^{\dagger}\right) .
$$

The expectation value of $x^{2}$ is then given by

$$
\left\langle x^{2}\right\rangle=\frac{\hbar}{2 m \gamma \beta_{+}}\left\langle\alpha_{+}^{\dagger} \alpha_{+}+\alpha_{+} \alpha_{+}^{\dagger}\right\rangle+\frac{\hbar}{2 m \gamma \beta_{+}}\left\langle\left(\alpha_{+}^{\dagger}\right)^{2}+\left(\alpha_{+}\right)^{2}\right\rangle+\frac{\hbar \beta_{-}}{2 m \gamma},
$$

where the cross-terms between plus and minus operators vanish because $\left\langle\alpha_{-}\right\rangle=\left\langle\alpha_{-}^{\dagger}\right\rangle=0$, and I use the relations $d_{ \pm}^{2} \lambda_{ \pm}^{2}=\left(\hbar / m \omega_{ \pm}\right)\left(\omega_{ \pm} / \mu_{ \pm}\right)=\hbar / m \gamma \beta_{ \pm}$. A similar calculation gives

$$
\left\langle y^{2}\right\rangle=\frac{\hbar \beta_{+}}{2 m \gamma}\left\langle\alpha_{+}^{\dagger} \alpha_{+}+\alpha_{+} \alpha_{+}^{\dagger}\right\rangle-\frac{\hbar \beta_{+}}{2 m \gamma}\left\langle\left(\alpha_{+}^{\dagger}\right)^{2}+\left(\alpha_{+}\right)^{2}\right\rangle+\frac{\hbar}{2 m \gamma \beta_{-}},
$$

and an appropriate linear combination leads to the quantity in Eq. (38). In this way, the desired LLL expectation value $\left\langle H_{0}\right\rangle$ has the simple form

$$
\left\langle H_{0}\right\rangle=\frac{1}{2} \hbar \omega_{-}-\frac{1}{4} \hbar \omega_{+}\left(\beta_{+} \beta_{-}+\frac{1}{\beta_{+} \beta_{-}}\right)+\frac{1}{2} m \gamma \omega_{+}\left(\beta_{+}\left\langle x^{2}\right\rangle+\frac{1}{\beta_{+}}\left\langle y^{2}\right\rangle\right) .
$$

For a symmetric trap with $\omega_{x}=\omega_{y}=\omega_{0}$, this result reduces to the familiar LLL expression $\left\langle H_{0}\right\rangle=\hbar \Omega+$ $m \omega_{0}\left(\omega_{0}-\Omega\right)\left\langle r^{2}\right\rangle$ [4, 8], where $r^{2}=x^{2}+y^{2}$.

In the case of a symmetric condensate, the special properties of the LLL states yield a simple well-known relation between the expectation value of the angular momentum and the mean-squared radius [4, 8]

$$
\frac{\left\langle L_{z}\right\rangle}{\hbar}=\frac{\left\langle r^{2}\right\rangle}{d_{0}^{2}}-1
$$

For an anisotropic condensate, an analogous result follows from the expectation value $\left\langle L_{z}\right\rangle=\left\langle x p_{y}-y p_{x}\right\rangle$ in a LLL state. Equations (19) for $x$ and $y$ and the corresponding relations for $p_{x}$ and $p_{y}$ lead to an expression involving the combinations $\left\langle\alpha_{+}^{\dagger} \alpha_{+}+\alpha_{+} \alpha_{+}^{\dagger}\right\rangle$ and $\left\langle\left(\alpha_{+}^{\dagger}\right)^{2}+\left(\alpha_{+}\right)^{2}\right\rangle$. Comparison with Eqs. (40) and (41) and use of Eq. (15) then yields the following generalization of (43)

$$
\frac{\left\langle L_{z}\right\rangle}{\hbar}=\frac{m \gamma}{2 \hbar}\left\langle x^{2}+y^{2}\right\rangle+\frac{m \omega_{-}}{2 \hbar}\left(\beta_{-}-\frac{1}{\beta_{-}}\right)\left\langle x^{2}-y^{2}\right\rangle-\frac{1}{2}\left(\beta_{-}+\frac{1}{\beta_{-}}\right) .
$$

For a symmetric trap $\left(\omega_{x}=\omega_{y}=\omega_{0}\right)$, it follows by inspection that this result has the correct limit (43), because $\gamma \rightarrow 2 \omega_{0},\left\langle y^{2}\right\rangle=\left\langle x^{2}\right\rangle$, and $\beta_{-} \rightarrow 1$.

\section{INTERACTING GAS IN A ROTATING ANISOTROPIC TRAP}

A dilute interacting Bose-Einstein condensate with $N$ particles in a trap is described by a condensate wave function $\psi$ that is here normalized to unity, with $\int d x d y|\psi|^{2}=1$. The Gross-Pitaevskii (GP) energy functional for this system involves both the noninteracting Hamiltonian $H_{0}$ from Eq. (1) and the interaction energy

$$
E[\psi]=\int d x d y\left(\psi^{*} H_{0} \psi+\frac{1}{2} g_{2 \mathrm{~d}} N|\psi|^{4}\right),
$$

where $g_{2 \mathrm{~d}}$ is a two-dimensional coupling constant with dimensions of energy times area. If the condensate is confined in a tight axial harmonic trap with oscillator length $d_{z}=\sqrt{\hbar /\left(m \omega_{z}\right)}$, then $g_{2 \mathrm{~d}}=\sqrt{8 \pi} \hbar^{2} a_{s} /\left(m d_{z}\right)$, where $a_{s}$ is the $s$-wave scattering length $[8,9,26]$. In contrast, for a condensate that is uniform in the $z$ direction with axial length $Z$, the analogous relation is $g_{2 \mathrm{~d}}=4 \pi \hbar^{2} a_{s} /(m Z)$. The Euler-Lagrange equation for the wave function is the stationary GP equation

$$
H_{0} \psi+g_{2 \mathrm{~d}} N|\psi|^{2} \psi=\mu \psi
$$

where the chemical potential $\mu$ is fixed by the normalization of $\psi$. 


\section{A. Lowest Landau-level limit for rapid rotation}

If the trap rotates rapidly, the condensate wave function $\psi$ can be approximated by a general LLL state $\psi_{L L L}=$ $\sum_{n} c_{n} \varphi_{n 0}$. In this case, the expectation value of $H_{0}$ simplifies considerably to Eq. (42). Correspondingly, the total energy functional takes the approximate form

$$
\begin{aligned}
E_{L L L}[\psi]= & \frac{1}{2} \hbar \omega_{-}-\frac{1}{4} \hbar \omega_{+}\left(\beta_{+} \beta_{-}+\frac{1}{\beta_{+} \beta_{-}}\right) \\
& +\int d x d y\left[\frac{1}{2} m \gamma \omega_{+}\left(\beta_{+} x^{2}+\frac{1}{\beta_{+}} y^{2}\right)|\psi|^{2}+\frac{1}{2} g_{2 \mathrm{~d}} N|\psi|^{4}\right],
\end{aligned}
$$

where I now follow [8] and omit the subscript $L L L$ on the condensate wave function.

If the restriction to the LLL states is ignored, the absolute minimum of $E_{L L L}[\psi]$ is found by varying $|\psi|^{2}$ subject solely to the normalization condition. The resulting approximate GP equation

$$
\frac{1}{2} m \gamma \omega_{+} \beta_{+} x^{2}+\frac{1}{2} m \gamma \omega_{+} \frac{1}{\beta_{+}} y^{2}+g_{2 \mathrm{~d}} N|\psi|^{2}=\mu
$$

implies an anisotropic density distribution

$$
\left|\psi_{\min }(x, y)\right|^{2}=\frac{\mu}{g_{2 \mathrm{~d}} N}\left(1-\frac{x^{2}}{R_{x}^{2}}-\frac{y^{2}}{R_{y}^{2}}\right)
$$

with characteristic condensate radii given by

$$
R_{x}^{2}=\frac{2 \mu}{m \gamma \omega_{+} \beta_{+}}, \quad R_{y}^{2}=\frac{2 \mu \beta_{+}}{m \gamma \omega_{+}}
$$

Note that the ratio $R_{y} / R_{x}=\beta_{+} \propto \omega_{+}$vanishes as $\Omega \rightarrow \omega_{x}$, but the behavior of the individual condensate radii requires a study of the chemical potential $\mu$.

As emphasized by various authors [4, 5, [6, 7, 8, 9], this density is very similar to the familiar Thomas-Fermi form for a nonrotating condensate in a stationary two-dimensional trap. In that case, the repulsive interactions expand the condensate and reduce the kinetic energy compared to the trap energy and the interaction energy. The situation here is very different, because the approximate LLL wave function explicitly incorporates the full single-particle Hamiltonian, including the kinetic energy; in this context, the appearance of the squared coordinates $x^{2}$ and $y^{2}$ arises from the special properties of the LLL states, specifically the result in Eq. (42).

The normalization condition for $|\psi|^{2}$ in (49) readily yields the condition

$$
\mu=\sqrt{\frac{m \gamma \omega_{+} g_{2 \mathrm{~d}} N}{\pi}}
$$

Note that the chemical potential $\mu$ vanishes proportional to $\sqrt{\omega_{+}} \propto \delta^{1 / 4}$ for a rapidly rotating anisotropic trap, where $\delta=1-\Omega / \omega_{x} \rightarrow 0$. Equation (49) then shows that the central density $\left|\psi_{\min }(0,0)\right|^{2}$ also has the same behavior in this limit.

A combination of Eqs. (501) and (51) gives the condensate radii

$$
R_{x}^{2}=\frac{2}{\beta_{+}} \sqrt{\frac{g_{2 \mathrm{~d}} N}{\pi m \gamma \omega_{+}}}, \quad R_{y}^{2}=2 \beta_{+} \sqrt{\frac{g_{2 \mathrm{~d}} N}{\pi m \gamma \omega_{+}}} .
$$

Correspondingly, the normalized minimizing density (49) becomes

$$
\left|\psi_{\min }(x, y)\right|^{2}=\frac{2}{\pi R_{x} R_{y}}\left(1-\frac{x^{2}}{R_{x}^{2}}-\frac{y^{2}}{R_{y}^{2}}\right) .
$$

Since $\omega_{+}$and $\beta_{+}$are both proportional to $\sqrt{\delta}$ as $\delta \rightarrow 0$ for fixed trap anisotropy, it is clear that $R_{x}^{2}$ grows like $\delta^{-3 / 4}$ and $R_{y}^{2}$ shrinks like $\delta^{1 / 4}$ for small $\delta$, which reflects the conservation of total number of particles. In particular, the total area $\pi R_{x} R_{y}$ diverges like $\omega_{+}^{-1 / 2} \propto \delta^{-1 / 4}$. This anisotropy of the minimizing $N$-body condensate in the limit $\delta \rightarrow 0$ is quite different from the anisotropy of the LLL single-particle ground state $\varphi_{00}$, where Eq. (28) shows that $a_{x}$ 
grows but $a_{y}$ approaches a constant as $\delta \rightarrow 0$. For the minimizing density (53), it is straightforward to evaluate the mean-squared displacements $\left\langle x^{2}\right\rangle=\frac{1}{6} R_{x}^{2}$ and $\left\langle y^{2}\right\rangle=\frac{1}{6} R_{y}^{2}$. The mean angular momentum in Eq. (44) then becomes

$$
\frac{\left\langle L_{z}\right\rangle}{\hbar}=\frac{m}{12 \hbar}\left(\omega_{+} \beta_{+}+\omega_{-} \beta_{-}\right) R_{x}^{2}+\frac{m}{12 \hbar}\left(\frac{\omega_{+}}{\beta_{+}}+\frac{\omega_{-}}{\beta_{-}}\right) R_{y}^{2}-\frac{1}{2}\left(\beta_{-}+\frac{1}{\beta_{-}}\right) .
$$

The condition $g_{2 \mathrm{~d}} n(0) \ll \hbar \omega_{-}$for the validity of the lowest Landau-level approximation can now be made explicit. Use of $g_{2 \mathrm{~d}} n(0) \approx 2 g_{2 \mathrm{~d}} N /\left(\pi R_{x} R_{y}\right)$ from (53), Eq. (52) for the condensate radii and the relation $g_{2 \mathrm{~d}} \approx 4 \pi \hbar^{2} a_{s} /(m Z)$ for a uniform condensate of thickness $Z$ yields

$$
2 \sqrt{\frac{\gamma \omega_{+}}{\omega_{-}^{2}} \frac{N a_{s}}{Z}} \ll 1
$$

Since $\omega_{+} \rightarrow 0$ and $\omega_{-}$remains nonzero for sufficiently rapid rotation $\left(\Omega \rightarrow \omega_{x}\right)$, this condition can always be satisfied. In the special case of a symmetric trap, the minimizing density has the isotropic form $[\underline{8}]$

$$
\left|\psi_{\min }(r)\right|^{2}=\frac{2}{\pi R_{0}^{2}}\left(1-\frac{r^{2}}{R_{0}^{2}}\right)
$$

with

$$
R_{0}^{2}=\left[\frac{2 g_{2 \mathrm{~d}} N}{\pi m \omega_{0}\left(\omega_{0}-\Omega\right)}\right]^{1 / 2} .
$$

This squared condensate radius diverges as $\Omega \rightarrow \omega_{0}$. Similarly, the absolute minimum of the LLL energy functional (47) for a symmetric trap becomes [8] $\left.E_{L L L}\right|_{\min }=\hbar \Omega+\frac{2}{3} m \omega_{0}^{2} R_{0}^{2}$. As a simple check, it is easy to verify that $-\left.\partial E_{L L L}\right|_{\min } / \partial \Omega=\hbar\left(\frac{1}{3} R_{0}^{2} / d_{0}^{2}-1\right)$; this result agrees with Eq. (43) because $\left\langle r^{2}\right\rangle=\left\langle x^{2}+y^{2}\right\rangle=\frac{1}{3} R_{0}^{2}$ in the symmetric limit.

\section{B. Density of vortices}

The general LLL state $\psi_{L L L}$ is a linear combination of the states $\varphi_{n 0}$. Apart from a normalization factor $1 / \sqrt{n !}$, each of these states is a polynomial $p_{n}(\zeta)$ multiplied by the ground state $\varphi_{00}$, where $\zeta$ from Eq. (31) is proportional to $x+i \beta_{-} y$. Thus the general LLL state also involves a polynomial in $\zeta$ that can formally be factorized to write

$$
\psi_{L L L} \propto \varphi_{00} \prod_{j}\left(\zeta-\zeta_{j}\right)
$$

The corresponding LLL particle density $n_{L L L}=\left|\psi_{L L L}\right|^{2}$ becomes

$$
n_{L L L} \propto\left|\varphi_{00}\right|^{2} \prod_{j}\left|\zeta-\zeta_{j}\right|^{2}
$$

Apart from an additive constant, the logarithm of this relation gives [4, 8, 27, 28]

$$
\begin{aligned}
\sum_{j} \ln \left|\left(x-x_{j}\right)^{2}+\beta_{-}^{2}\left(y-y_{j}\right)^{2}\right| & =\frac{x^{2}}{a_{x}^{2}}+\frac{y^{2}}{a_{y}^{2}}+\ln n_{L L L}(\boldsymbol{r}) \\
& =\frac{1}{a_{x}^{2}}\left(x^{2}+\frac{\beta_{-} y^{2}}{\beta_{+}}\right)+\ln n_{L L L}(\boldsymbol{r}) .
\end{aligned}
$$

Here I use Eqs. (28) and (29) for the anisotropic ground state and note that $a_{y}^{2} \beta_{-}=a_{x}^{2} \beta_{+}$.

To include the anisotropy of the complex variable $\zeta \propto x+i \beta_{-} y$, it is convenient to introduce the rescaled variables $x^{\prime}=x$ and $y^{\prime}=\beta_{-} y$. Application of the rescaled Laplacian $\nabla^{\prime 2}=\partial^{2} / \partial x^{\prime 2}+\partial^{2} / \partial y^{\prime 2}$ readily gives

$$
\sum_{j} \nabla^{\prime 2} \ln \left|\boldsymbol{r}^{\prime}-\boldsymbol{r}_{j}^{\prime}\right|^{2}=\frac{2}{a_{x}^{2}}\left(1+\frac{1}{\beta_{+} \beta_{-}}\right)+\nabla^{\prime 2} \ln n_{L L L}(\boldsymbol{r}) .
$$


Since $\nabla^{\prime 2} \ln \left|\boldsymbol{r}^{\prime}\right|^{2}=4 \pi \delta\left(x^{\prime}\right) \delta\left(y^{\prime}\right)=\left(4 \pi / \beta_{-}\right) \delta(x) \delta(y)$, this relation implies

$$
n_{v}(\boldsymbol{r})=\frac{m \gamma}{2 \pi \hbar}+\frac{\beta_{-}}{4 \pi} \nabla^{\prime 2} \ln n_{L L L}(\boldsymbol{r})
$$

where $n_{v}(\boldsymbol{r})=\sum_{j} \delta^{(2)}\left(\boldsymbol{r}-\boldsymbol{r}_{j}\right)$ is the two-dimensional vortex density, and I have again used Eq. (28). For a rapid rotation speed $\Omega \lesssim \omega_{x} \leq \omega_{y}$ and any trap anisotropy, the frequency $\gamma=\left(\omega_{-}^{2}-\omega_{+}^{2}\right) /(2 \Omega)$ is given in terms of elementary expressions from Eqs. (3) and (4), as shown in Fig. 1(a) for a typical anisotropy ratio $\omega_{y} / \omega_{x}=1.2$.

To estimate the vortex density $n_{v}$ in Eq. (62), assume that the equilibrium particle density $n_{L L L}$ is that given by the absolute minimum solution in Eq. (49), with $n_{L L L}(\boldsymbol{r}) \propto 1-x^{2} / R_{x}^{2}-y^{2} / R_{y}^{2}$. A straightforward calculation then yields

$$
\begin{array}{r}
n_{v}(x, y) \approx \frac{m \gamma}{2 \pi \hbar}-\frac{\beta_{-}}{2 \pi\left(1-x^{2} / R_{x}^{2}-y^{2} / R_{y}^{2}\right)^{2}}\left[\frac{1}{R_{x}^{2}}+\frac{1}{\beta_{-}^{2} R_{y}^{2}}\right. \\
\left.+\left(\frac{y^{2}}{R_{y}^{2}}-\frac{x^{2}}{R_{x}^{2}}\right)\left(\frac{1}{\beta_{-}^{2} R_{y}^{2}}-\frac{1}{R_{x}^{2}}\right)\right] .
\end{array}
$$

For an isotropic trap $\left(\omega_{x}=\omega_{y}=\omega_{0}\right)$, this expression reduces to the well-known axisymmetric result [7, 27, 28]

$$
n_{v}(r) \approx \frac{m \omega_{0}}{\pi \hbar}-\frac{1}{\pi R_{0}^{2}} \frac{1}{\left(1-r^{2} / R_{0}^{2}\right)^{2}},
$$

where $R_{0}^{2}$ is given in Eq. (57).

Comparison of these two expressions shows some interesting differences:

1. For fixed angular velocity $\Omega \lesssim \omega_{0}$, the vortex density [64) in a rapidly rotating symmetric trap decreases gradually and isotropically away from the center of the condensate. Such behavior has been observed at lower angular velocities in the mean-field Thomas-Fermi regime [29]. In contrast, the general expression for the vortex density in Eq. (63) displays explicit anisotropy between $x$ and $y$.

2. To sharpen this analysis, it is convenient to focus on the central vortex density

$$
n_{v}(0) \approx \frac{m \gamma}{2 \pi \hbar}-\frac{1}{2 \pi}\left(\frac{\beta_{-}}{R_{x}^{2}}+\frac{1}{\beta_{-} R_{y}^{2}}\right) .
$$

For a symmetric trap, Eq. (64) shows that $n_{v}(0)$ increases monotonically with increasing $\Omega \leq \omega_{0}$, because the mean condensate radius $R_{0}$ grows in the same limit. For an anisotropic trap, in contrast, the frequency $\gamma$ and $R_{x}^{-2}$ both decrease with increasing $\Omega$, whereas $R_{y}^{-2}$ increases. Thus the combined effect of anisotropy and rapid rotation can, in principle, yield a central vortex density $n_{v}(0)$ that varies non-monotonically with increasing $\Omega$, as can be seen for typical numerical examples.

It is not clear whether either of these behaviors would be observable in practice.

The present discussion has focused on the "macroscopic" parabolic density profile that provides an absolute minimum of the energy in the rotating frame, ignoring the local distortions associated with the vortex cores. In practice, these phenomena have very different length scales: in the rapidly rotating limit, the vortex core and the intervortex separation are both of order $\sqrt{\hbar /(m \Omega)}$, whereas the condensate radii $R_{x}$ and $R_{y}$ are generally much larger. Thus it is possible to treat the condensate density as locally uniform over the size of an individual vortex. If the vortex lattice is treated as triangular and unbounded, this analysis yields a simple renormalization [5, 7, 8, 9, 30] of the interaction constant $g_{2 \mathrm{~d}} \rightarrow b g_{2 \mathrm{~d}}$, where $b \approx 1.1596$ is the numerical value for a triangular Abrikosov vortex lattice [31]. Apart from this rescaling of the interaction parameter, the description remains essentially unchanged.

\section{CONCLUSIONS AND DISCUSSION}

This work has examined the behavior of a two-dimensional Bose-Einstein condensate in an anisotropic harmonic trap (with general trap frequencies $\omega_{x} \leq \omega_{u}$ ) that rotates rapidly at an angular velocity $\Omega \lesssim \omega_{x}$. The single-particle Hamiltonian in Eq. (1) is exactly soluble 10, 11, 12], although the detailed form of the low-lying quantum-mechanical states in Eq. (30) for an arbitrary anisotropy has apparently not appeared previously. 
The ground-state wave function $\varphi_{00}(x, y)$ in Eq. (27) is an anisotropic Gaussian with $\Omega$-dependent characteristic lengths $a_{x}$ and $a_{y}$ given in (28). In addition, the ground state has a phase proportional to $x y$ [23, 24]. In the quantum problem, this behavior reflects the classical velocity potential for an irrotational (vortex-free) fluid confined in a rotating elliptical boundary $[22]$.

Similar to the case of a rapidly rotating symmetric condensate, the two eigenfrequencies $\omega_{ \pm}$in Eq. (2) for a rapidly rotating anisotropic condensate have very different magnitudes: $\omega_{+}$vanishes as $\Omega \rightarrow \omega_{x}$ (the smaller of the two trap frequencies), but $\omega_{-}$remains finite in this same limit. If the mean interaction energy $\sim g_{2 \mathrm{~d}} n(0)$ is small compared to the gap $\hbar \omega_{-}$between the ground state and the first excited Landau level, then the system can be described with the set of lowest Landau-level states $\varphi_{n 0}(x, y)$, where $n$ is a non-negative integer describing the number of plus quanta, each with the small energy $\hbar \omega_{+}$. Apart from the Gaussian factor $\varphi_{00}(x, y)$, these states involve a polynomial $p_{n}(\zeta)$ of order $n$, where $\zeta$ single complex variable proportional to $x+i \beta_{-} y$ and $\beta_{-} \leq 1$ is a positive constant for any $0<\Omega \leq \omega_{x}$.

Within the set of lowest Landau-level states $\psi_{L L L}=\sum_{n} c_{n} \varphi_{n 0}$, the expectation value $\left\langle H_{0}\right\rangle$ of the single-particle Hamiltonian can be reduced to an anisotropic linear combination of $\left\langle x^{2}\right\rangle$ and $\left\langle y^{2}\right\rangle$. An anisotropic Thomas-Fermi-like density $\left|\psi_{\min }(x, y)\right|^{2} \propto 1-x^{2} / R_{x}^{2}-y^{2} / R_{y}^{2}$ provides an absolute lower bound for the total energy of the interacting system. Here the condensate radii $R_{x}$ and $R_{y}$ are given in Eq. (52); $R_{x}^{2}$ grows and $R_{y}^{2}$ shrinks as $\Omega \rightarrow \omega_{x}$, while the area $\pi R_{x} R_{y}$ of the elliptical condensate grows slowly in the same limit.

As emphasized by Ho [4], the particle density $n_{L L L}(x, y)=\left|\psi_{L L L}(x, y)\right|^{2}$ in the lowest Landau-level limit also contains a description of the associated vortex density $n_{v}(x, y)$. This situation arises because the general linear combination of lowest Landau-level states is essentially a polynomial $P(\zeta)$ in the single complex variable $\zeta \propto x+i \beta \_y$, and the zeros $\left\{\zeta_{j}\right\}$ of $P(\zeta)$ represent the positions of the vortices in the $x y$ plane. The actual particle density $n_{L L L}$ has small-scale structure arising from the vortex cores, superposed on the parabolic global shape. If this fine-grain aspect is ignored, the resulting vortex density follows immediately in Eq. (63).

This work raises several interesting questions:

1. Feynman's familiar expression for the mean vortex density $n_{F}=m \Omega /(\pi \hbar)$ in a large symmetric rotating condensate requires modification for an anisotropic rotating condensate because of the irrotational flow induced by the rotating walls. Specifically, this irrotational flow contributes to the total angular momentum and thus lowers the energy $E^{\prime}=E-\Omega L_{z}$ in the rotating frame, delaying the first transition to a state containing a single quantized vortex 23. Indeed, the measured critical angular velocity $\Omega_{c}$ for the appearance of the first vortex in a rigid elliptical or rectangular cylinder containing uniform superfluid ${ }^{4}$ He exceeds that for a circular cylinder by a factor that increases with increasing anisotropy [32], confirming the theoretical prediction. A similar but more complicated situation occurs for slowly rotating weakly interacting anisotropic Bose-Einstein condensates [11]. Although the analogous situation with many vortices has not been studied in detail, the increased critical angular velocity for the appearance of the first vortex suggests that the mean vortex density at moderate rotation speeds in an anisotropic trap is likely to be smaller than in a corresponding symmetric trap.

In contrast, for a rapidly rotating anisotropic condensate, Eq. (65) gives the central density $n_{v}(0) \approx m \gamma /(2 \pi \hbar)$, apart from finite-size corrections associated with the condensate radii. This value of $n_{v}(0)$ typically exceeds $n_{F}$, because $\gamma /(2 \Omega) \rightarrow\left(3 \omega_{x}^{2}+\omega_{y}^{2}\right) /\left(4 \omega_{x}^{2}\right)>1$ in the limit $\Omega \rightarrow \omega_{x}$. The physical basis for such enhanced vortex density is not immediately obvious, and additional clarification would be desirable.

2. The present LLL approach ignores the fine-grain structure of the vortex lattice (apart from the renormalization of the interaction parameter $g_{2 \mathrm{~d}}$ by the "Abrikosov parameter" $b \sim 1.16$ ) [5, 7, 8, 9, 30]. In the special case of a nearly symmetric trap rotating close to the limit of instability, Oktel 12] finds that the vortex lattice remains almost exactly triangular, with the principal lattice planes aligned with the direction of weak trap confinement. It is not clear whether this situation holds for arbitrary anisotropy and less extreme rotation speeds.

3. As $\Omega$ approaches the weak confining frequency $\omega_{x}$, the condensate becomes essentially one dimensional and the vortices must then rearrange themselves to form a one-dimensional structure [20, 21]. Ultimately, this behavior may be pre-empted by some sort of transition to a correlated (nonsuperfluid) state, as has been predicted for a symmetric trap [33].

It will be interesting to investigate these various questions in detail.

\section{Acknowledgments}

I am grateful to G. Bertsch for early guidance on the literature about rotating deformed nuclei and rotating 
anisotropic oscillator potentials.

[1] K.W. Madison, F. Chevy, W. Wohlleben, and J. Dalibard, Phys. Rev. Lett. 84, 806 (2000).

[2] J.R. Abo-Shaeer, C. Raman, J.M. Vogels, and W. Ketterle, Science 292, 476 (2001).

[3] P.C. Haljan, I. Coddington, P. Engels, and E.A. Cornell, Phys. Rev. Lett. 87, 210403 (2001).

[4] T.L. Ho, Phys. Rev. Lett. 87, 060403 (2001).

[5] G. Baym and C.J. Pethick, Phys. Rev. A 69, 043619 (2004).

[6] N.R. Cooper, S. Komineas, and N. Read, Phys. Rev. A 70, 033604 (2004).

[7] G. Watanabe, G. Baym, and C.J. Pethick, Phys. Rev. Lett. 93, 190401 (2004).

[8] A. Aftalion, X. Blanc, and J. Dalibard, Phys. Rev. A 71, 023611 (2005).

[9] G. Watanabe, S.A. Gifford, G. Baym, and C.J. Pethick, cond-mat/0606151

[10] J.G. Valatin, Proc. Roy. Soc. 238, 132 (1956). Note that Valatin uses a coordinate system that rotates around $\hat{\boldsymbol{x}}$, so that many of the detailed expressions here differ from his.

[11] M. Linn, M. Niemeyer, and A.L. Fetter, Phys. Rev. A 64, 023602 (2001).

[12] M.Ö. Oktel, Phys. Rev. A 69, 023618 (2004).

[13] H. Lamb, Dynamics, 2nd edition (Cambridge University Press, Cambridge, 1923), pp. 91-92.

[14] L. Pitaevskii and S. Stringari, Bose-Einstein Condensation (Clarendon Press, Oxford, 2003), sec. 14.5.

[15] See, for example, R. Shankar, Principles of Quantum Mechanics, 2nd edition (Plenum Press, New York and London, 1994), sec. 7.4 .

[16] N.N. Bogoliubov, J. Phys. USSR 11, 23 (1947).

[17] See, for example, A.L. Fetter and J.D. Walecka, Theoretical Mechanics of Particles and Continua (Dover Publications, Inc., Mineola, NY, 2003), chap. 6. I follow Valatin in using $S$ for the generating function involving the old and new coordinates, instead of the more usual $F$.

[18] C. Cohen-Tannoudji, B. Diu, and F. Laloë, Quantum Mechanics (Wiley, New York, 1977), vol. I, pp. 733-738. These authors use the French abbreviations " $\mathrm{d}$ " and "g" to denote right- and left-circular polarization, instead of the present "plus" and "minus".

[19] M. Linn and A.L. Fetter, Phys. Rev. A 60, 4910 (1999).

[20] S. Sinha and G.V. Shlyapnikov, Phys. Rev. Lett. 94, 150401 (2005).

[21] P. Sánchez-Lotero and J.J. Palacios, Phys. Rev. A 72, 043613 (2005).

[22] H. Lamb, Hydrodynamics (Dover Publications, New York, 1945), 6th edition, pp. 86-88.

[23] A.L. Fetter, J. Low Temp. Phys. 16, 533 (1974).

[24] L. Pitaevskii and S. Stringari, Bose-Einstein Condensation (Clarendon Press, Oxford, 2003), sec. 14.2.

[25] See, for example, the Wolfram web site http://functions.wolfram.com/Polynomials/HermiteH

[26] A.L. Fetter, Phys. Rev. A 68, 063617 (2003).

[27] D.E. Sheehy and L. Radzihovsky, Phys. Rev. A 70, 051602(R) (2004).

[28] D.E. Sheehy and L. Radzihovsky, Phys. Rev. A 70, 063620 (2004).

[29] I. Coddington, P.C. Haljan, P. Engels, V. Schweikhard, S. Tung, and E.A. Cornell, Phys. Rev. A 70, 063607 (2004).

[30] J. Sinova, C.B. Hanna, and A.H. MacDonald, Phys. Rev. Lett. 89, 030403 (2002).

[31] W.H. Kleiner, L.M. Roth, and S.H. Autler, Phys. Rev. 133, A1226 (1964).

[32] K. DeConde and R.E. Packard, Phys. Rev. Lett. 35, 732 (1975).

[33] N.R. Cooper, N.K. Wilkin, and J.M.F. Gunn, Phys. Rev. Lett. 87, 120405 (2001). 